\title{
SHIELDING EFFECTIVENESS OF ANISOTROPIC MATERIALS: HOW TO MEASURE?
}

\author{
Johan Catrysse*, Filip Vanhee, Tim Claeys, Andy Degraeve, Davy Pissoort \\ ReMI, Reliability in Mechatronics and ICT \\ KU Leuven, Technology Campus Oostende \\ Zeedijk 101, B 8400 Oostende (Belgium) \\ MICAS, ESAT, KU Leuven (Leuven - Belgium)
}

\begin{abstract}
In a number of applications, due to concerns such as weight and flexibility, shielding materials are used with anisotropic materials. Typically, in the case of some conductive textile materials, conductive yarns are only used in one direction (warp). Other materials will have different conductivity as a function of the direction, such as deployed metal grids. In this paper, measured Shielding Effectiveness SE results obtained by using different measuring setups are compared and discussed.
\end{abstract}

Keywords: shielding materials, measurements

\section{INTRODUCTION}

In a number of applications, due to concerns as weight and flexibility, shielding materials are used with anisotropic materials. Typically, in the case of some conductive textile materials, conductive yarns are only used in one direction (warp).

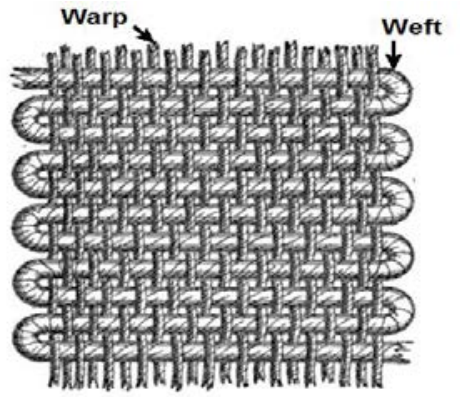

Figure 1. Warp and weft in a woven textile sheet

Other materials will have different conductivity as a function of the direction, such as deployed metal grids and meshes.

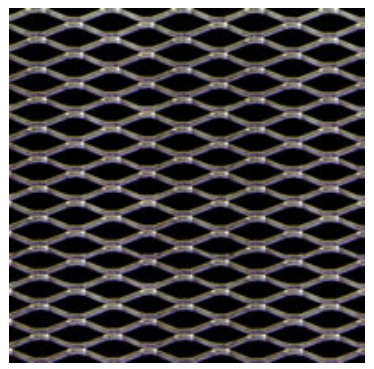

Figure 2. Example of deployed metal

In this paper, measured SE results obtained by using different measuring setups are compared and discussed.

\section{MEASURING METHODS}

Actually, a number of possible measuring methods are proposed and discussed in literature [1] - [5]. A number of them are standardized and based on a modified IEEE Std 299 (formerly MIL STD 285) methodology [1] or a coaxial method based on ASTM D4935 [2].

The actual standard IEEE Std 299 is covering the characterization of shielded enclosures to $18 \mathrm{GHz}$. By making a hole in one of the walls of an enclosure and covering this hole with the material under test, the SE of the material can be measured. The general setup for the IEEE Std 299 methodology [1] and similar configurations is shown in figure 3 .

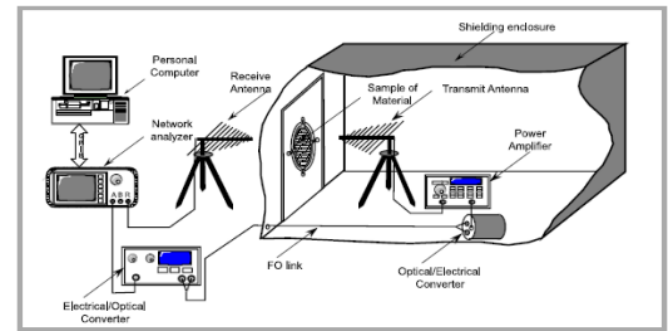

Figure 3. Global view of IEEE Std 299 setup and similar configurations

Another well established method for the characterization of conductive planar materials is the coaxial method, as described in the standard ASTM D4935 [2]. Although originally intended for the frequency range from DC to $1 \mathrm{GHz}$, extended frequency ranges are reported in literature [5] - [6].

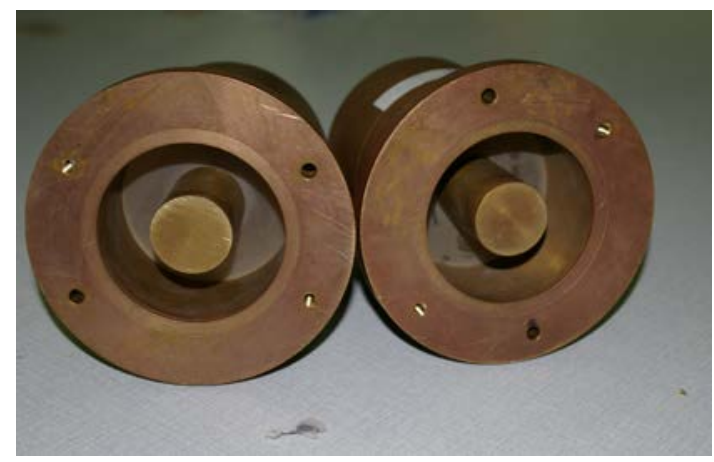

Figure 4 . Example of the two halfs of an ASTM D4935 fixture 
Other methods reported in literature are not standardized, but very similar to the ASTM D4935 method. They are based on a TEM cell with a rectangular cross section and are claimed to be able to show the discrimination in SE as a function of the polarization for materials with directional properties [3] - [4].
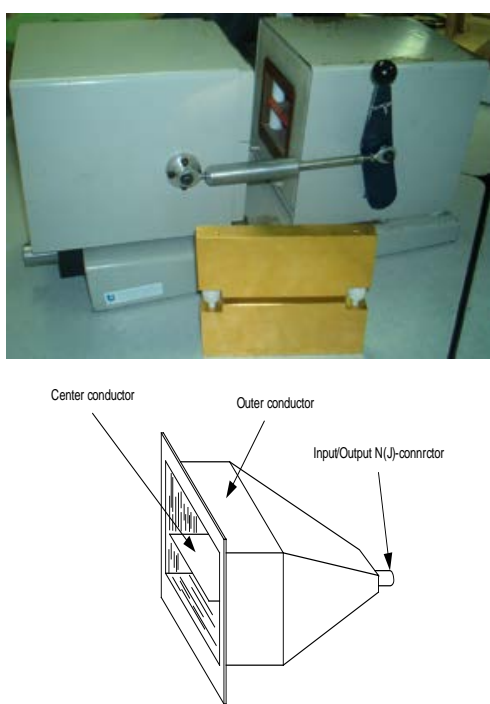

Figure 5. Example of the TEMt-cell, similar to ASTM D4935, but with a rectangular cross section.

\section{MEASURED SHIELDING EFFECTIVENESS}

The next series of graphs shows two sets of measured SE values of a isotropic (figure 6) and a nearly isotropic (figure 7) material, using three measurement setups: IEEE Std 299 using a logper antenna, ASTM D4935 and TEMt-cell.

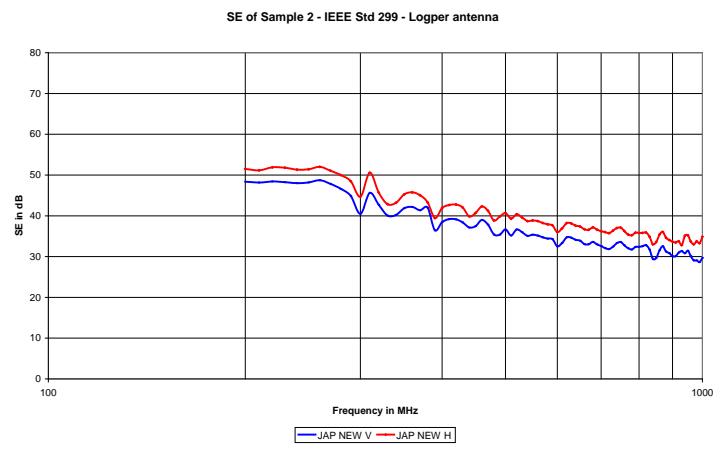

SE van Sample 2 - astm D4935

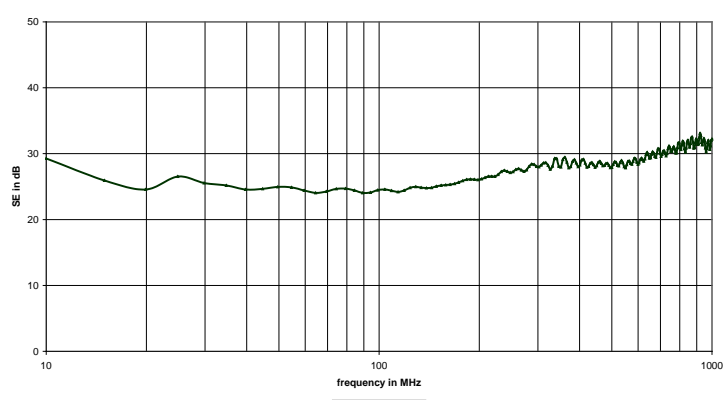

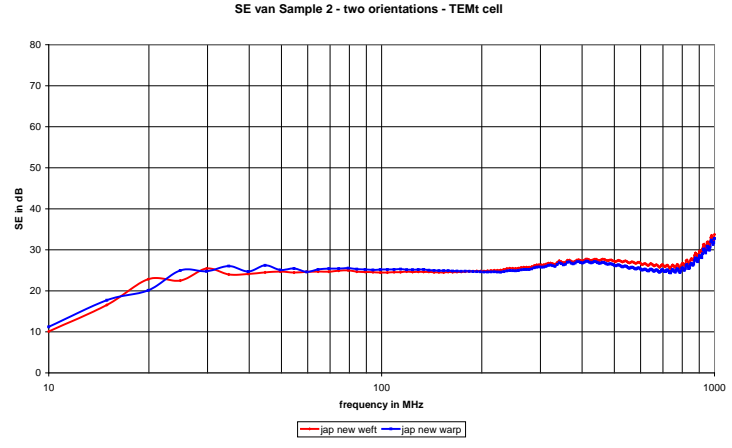

Figure 6. Measured SE of a isotropic sample, using IEEE Std 299, ASTM D4935 and TEMt-cell methods

Both ASTM D4935 and TEMt-cell tend to produce similar results. Concerning the IEEE Std 299 cell, it must be noted that the typical size of the samples is $50 \mathrm{~cm} \times 50 \mathrm{~cm}$, so that correct interpretation of the obtained SE is only valid from about $200 \mathrm{MHz}$ on. A small difference in SE is observed for the IEEE Std 299 method as a function of the polarization or the orientation of the measuring antennas.
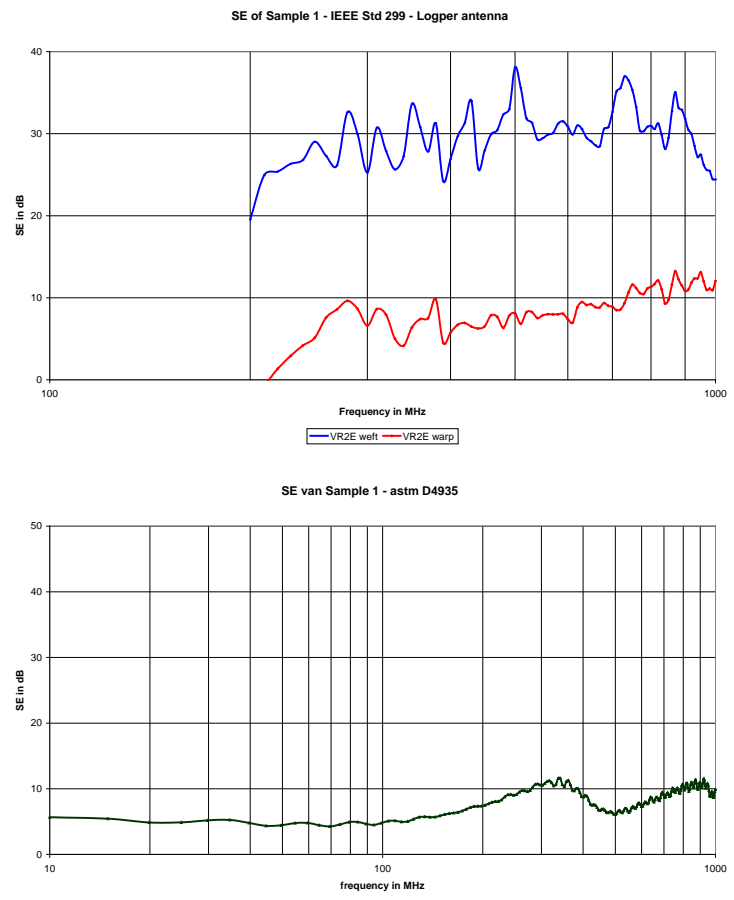

$=$ W2e-astm

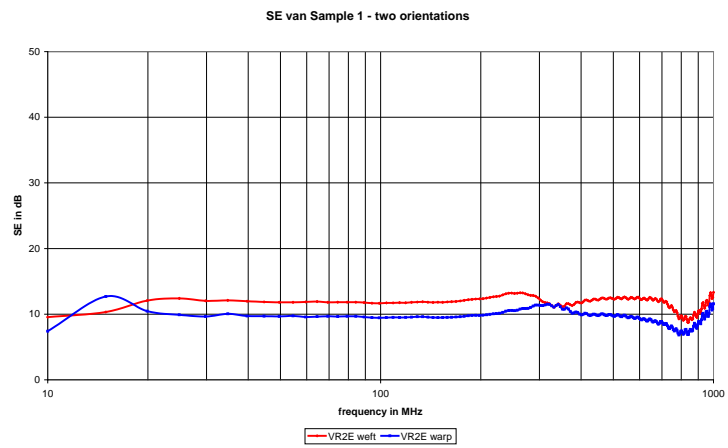

Figure 7. Measured SE of an a nearly isotropic sample, using IEEE Std 299, ASTM D4935 and TEMt-cell methods 
The measured SE of a nearly isotropic material is reported in figure 7 . It consists of a woven textile with identical yarns for both weft and warp, but the stress on both yarns is different due to the weaving procedure. Some anisotropic-like characteristics are clearly seen from the IEEE Std 299 method, but are completely disappearing for the ASTM D4935 method, and partly for the TEMt-cell.

Due to the circular symmetry of the ASTM D4935 fixture, polarization effect can no more be observed and the SE value becomes also very low. Due to the rectangular cross section of the TEMt-cell, it was supposed that an electric field distribution with a dominant polarization should occur inside the cell. However, in this case, there is nearly no discrimination about the anisotropic characteristics of the sample, and the measured SE tends to be significantly higher than the ASTM one.

\section{ELECTRIC FIELD DISTRIBUTIONS}

In order to understand the discussion, the electric field distribution of three measuring setups is given in the figure 8, as reported in references [7] - [8]:
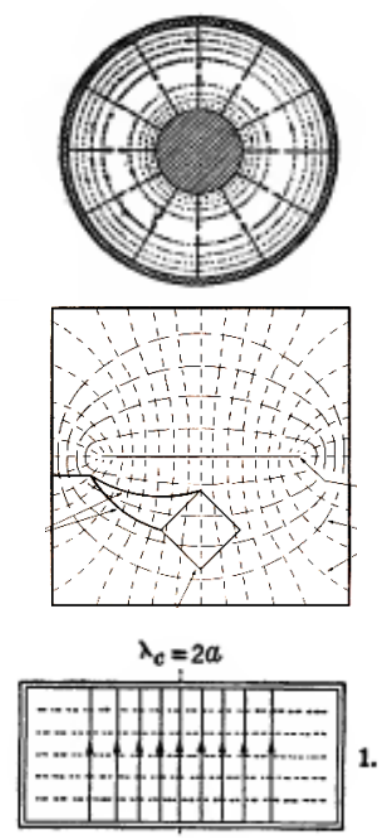

Figure 8. Electric field distribution inside the ASTM D4935 cell, Temt-cell and Horn antenna [7] - [8]

It can be concluded that a horn antenna is providing a measuring environment with well-defined polarization, but that both ASTM D4935 cell and TEMt cell do not have a unique "one direction" polarization. It follows that anisotropic materials will show different SE behaviour when using test cells of different types and cross section.

Measurements performed using the ASTM D4935 fixture and the TEMt-cell are in the frequency range $10 \mathrm{MHz}$ - $1 \mathrm{GHz}$, but some measurements using horn antennas are in the frequency range from $1 \mathrm{GHz}$ up to $10 \mathrm{GHz}$.
In order to correlate results from both frequency ranges, measurements are performed over the global frequency range using the IEEE Std 299 setup with a logper and a horn antenna.

\section{SE OF AN ANISOTROPIC TEXTILE}

An anisotropic woven textile, with even only a few $\%$ of stainless steel fibres in the conductive weft yarn has been measured using a number of fixtures and are given in figures 9 and 10 .
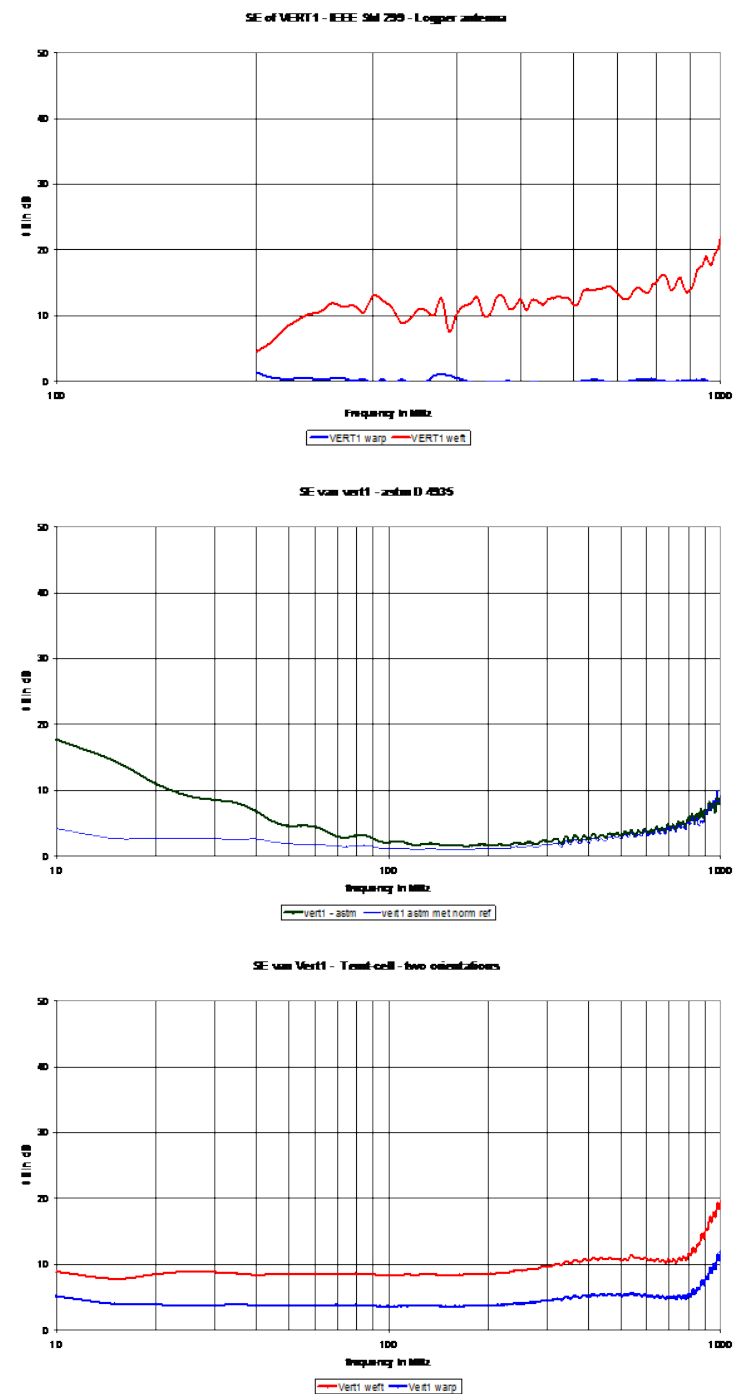

Figure 9. SE of an anisotropic woven textile, IEEE Std 299 (upper), ASTM D4935 (middle) and TEMt-cell (lower)

The fact that there is only a conductive yarn in the weft is clearly seen from the IEEE Std 299 SE result, where in the warp direction, no SE at all is observed. Different SE values are obtained for the weft or the warp when inserted in the TEMt-cell. But the $0 \mathrm{~dB}$ SE of the warp direction is not observed. Concerning the ASTM D4935 results, the blue line has been obtained by the correct application of the standard procedures, by inserting a small piece of the sample in between both inner conductors for performing the reference measurement, while the black line shows the SE with a wrong continuous inner conductor reference. 

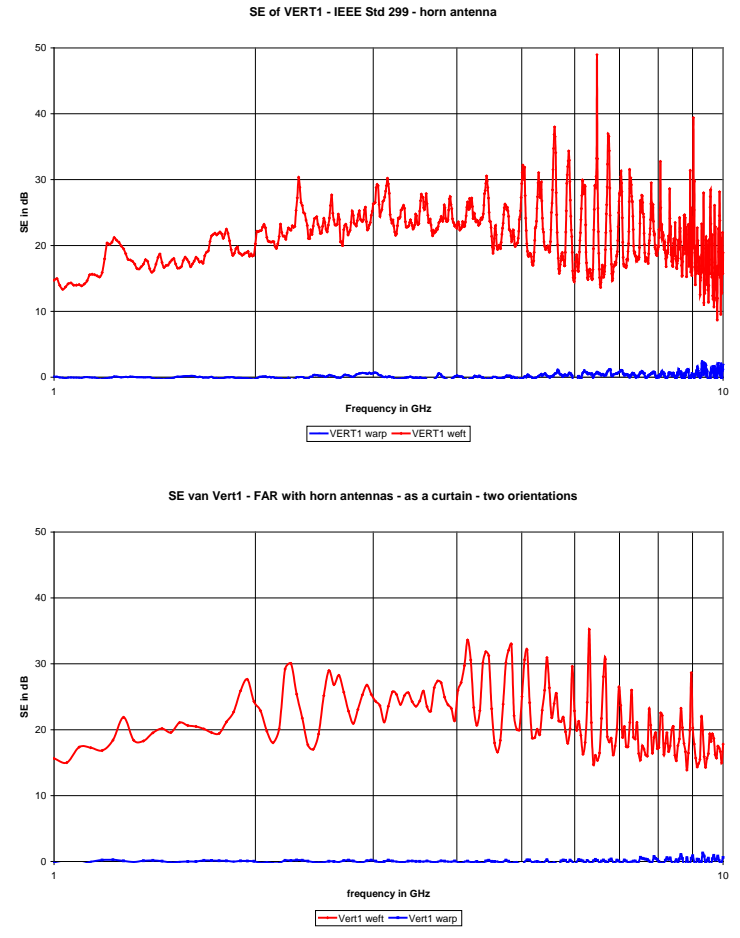

Figure 10. SE between $1 \mathrm{GHz}$ - $10 \mathrm{GHz}$, using the IEEE Std 299 with a horn antenna, and a free space setup with horn antennas

First of all, similar SE results for both setups are observed. Secondly, the discrimination between the weft and the warp directions is very clear, given also the zero SE for the warp direction. And finally, the continuity between both IEEE Std 299 frequency ranges is shown.

The free space setup consists of a curtain-like large sample (2.5 x $2.5 \mathrm{~m})$, hanging in the free space of a FAR and with two horn antennas. Distances between sample and antennas is identical to the IEEE Std 299 method.

The setup is shown in figure 11 .

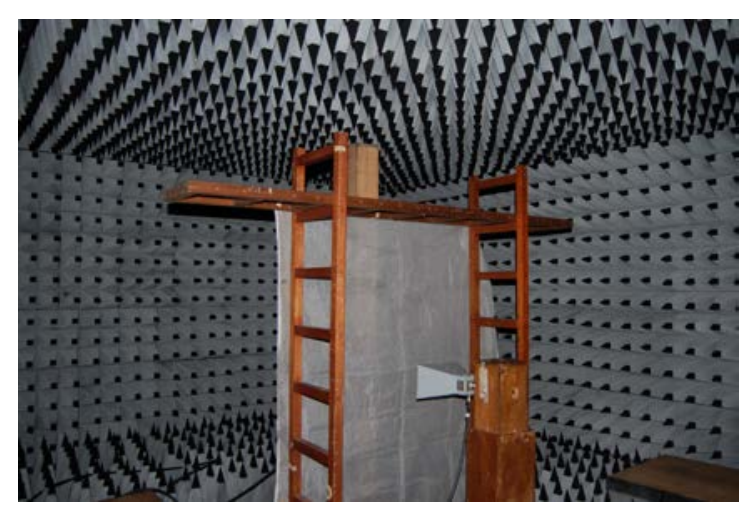

Figure 11. Free space "curtain-like" setup in a FAR

Another test was performed using a small handmade sample $(12 \mathrm{~cm} \times 12 \mathrm{~cm})$ with wires $5 \mathrm{~mm}$ apart, glued on a wooden substrate, as shown in figure 12 . SE results (figure 14a) obtained from the TEMt-cell show different SE values for two polarizations, But the $0 \mathrm{~dB} \mathrm{SE}$ of one direction of the wires is not observed. A strong resonance is observed at the higher frequencies

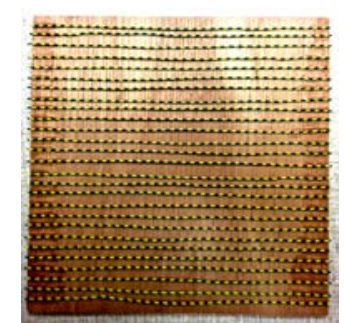

Figure 12. Hand-made sample with parallel wires at $5 \mathrm{~mm}$

A cell consisting of two horn antennas, clamped together, has been used for the higher frequency range [9].

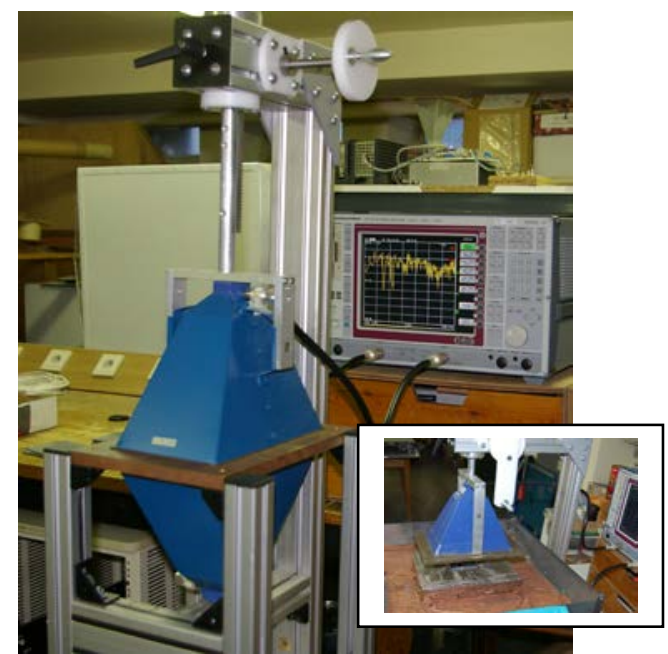

Figre 13. Double horn cell for SE measurement above $1 \mathrm{GHz}$

Measured SE shows in the lower $\mathrm{GHz}$ region the clear discrimination for two polarizations with respect to the direction of the parallel wires.
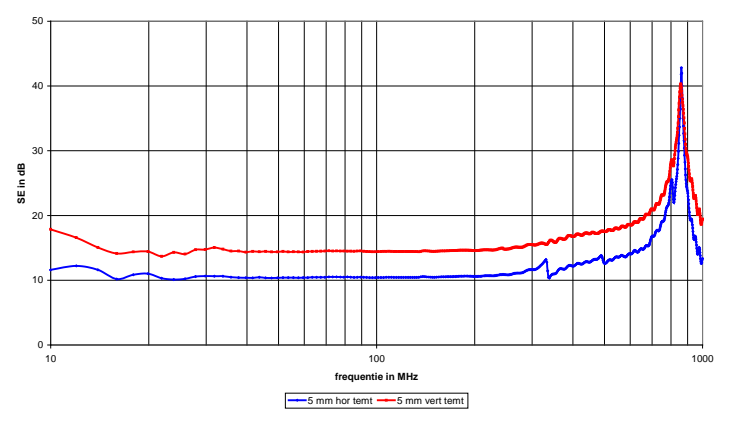

SE of wires, spaced at $5 \mathrm{~mm}$ - Horn cell

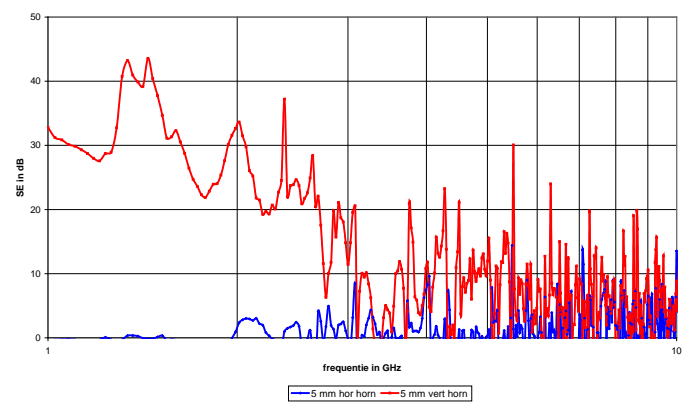

Figure 14. SE for parallel wires at $5 \mathrm{~mm}$, (a) from TEMt-cell (upper) and (b) a horn cell (lower) 


\section{SOME PRELIMINARY SIMULATIONS}

Starting from this small hand-made wire sample and the measured SE results from the TEMt-cell, a first set of preliminary simulations have been performed by a full wave FDTD simulation as included in Keysight Technologies EMPro.
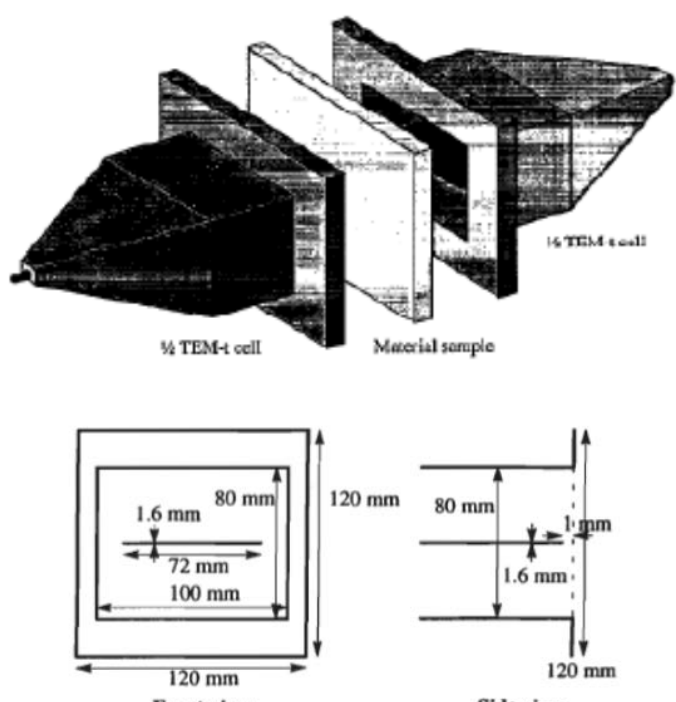

Erent view

Side view

Figure 15. TEMt-cell with sample (upper) and detailed cross sections (lower) of the TEMt-cell for the simulations

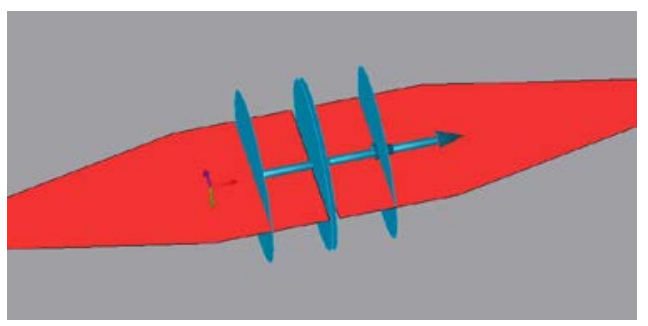

Figure 16. Interrupted septum of the TEMt-cell, and the sensor planes for simulation of the E field distribution

A first simulation dealt with two isotropic and homogeneous materials, with different conductivities $\sigma$ (5 and 100), in order to check the validity of the model used. A difference of about $25 \mathrm{~dB}$ SE should be observed for both materials, and for the sample with the higher conductivity, a slope of - $20 \mathrm{~dB} /$ decade should occur at the lower frequency range.

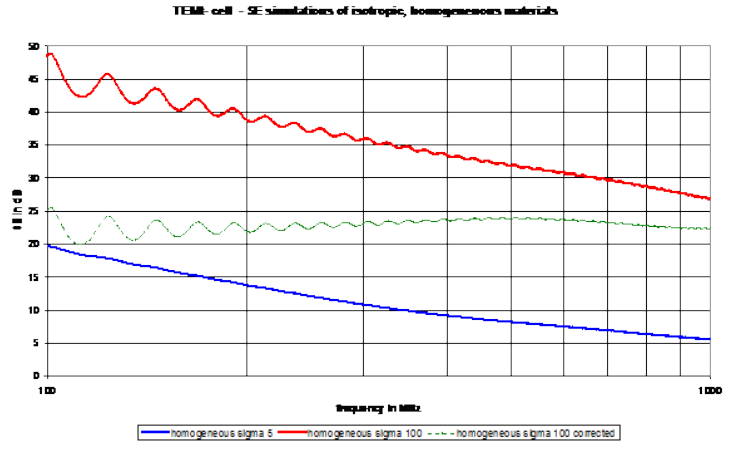

Figure 17. Simulation of two isotropic homogeneous samples in the TEMt-cell
As the field distribution is not homogeneous inside the TEMt-cell (figure 8), a first idea was to split the E-field in $\mathrm{x}$ and $\mathrm{y}$ components, and allocate a different attenuation to each of them, resulting from plane wave measurements (see figures 9 and 10). This field distribution is also seen in figure 18 .

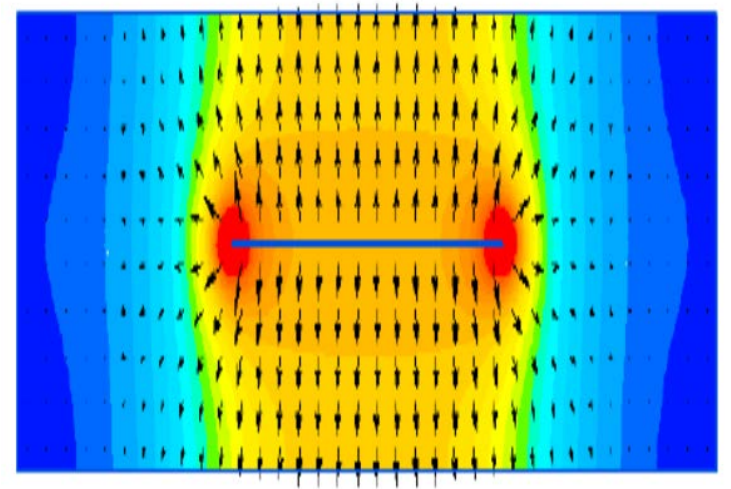

Figure 18. Orientation of the E-field in the TEMt-cell

Referring to the measured results from a sample as reported in figure 9 and 10, an attenuation of $20 \mathrm{~dB}$ was assumed for one component and $0 \mathrm{~dB}$ for the other one. Depending on the application of these figures to the $(\mathrm{x}, \mathrm{y})$ or $(\mathrm{y}, \mathrm{x})$ components, a resulting $\mathrm{SE}$ of $10.5 \mathrm{~dB}$ and $2 \mathrm{~dB}$ were obtained. This does not correlate with the measured SE values as given in figure 9, nor with measured TEMt-cell results as found for the hand-made sample in figure 14 (upper).

A full 3D field simulation of the TEMt-cell and a sample with parallel wires at $4 \mathrm{~mm}$ distance shows between $100 \mathrm{MHz}-1 \mathrm{GHz}$ a similar characteristic (figure 19) as the measured one of figure 14a.

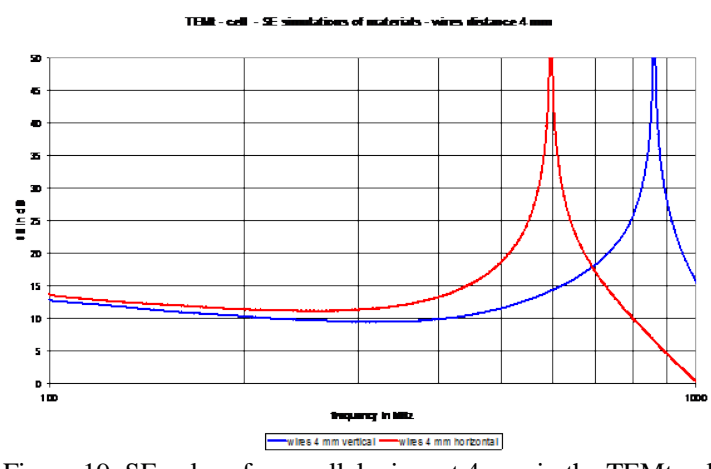

Figure 19. SE values for parallel wires at $4 \mathrm{~mm}$ in the TEMt-cell by $3 \mathrm{D}$ simulation

By looking into more detail to the field distributions inside the TEMt-cell, it must be concluded that the insertion of anisotropic materials will introduce very non-homogeneous field patterns beyond the wired sample.

It looks like a position dependant attenuation is occurring, so that a simple calculation model, assuming a homogeneous attenuation over the whole cross section of the TEMt-cell, does not work properly for (nearly) anisotropic materials.

A set of examples of these field distributions is given in figures 20 and 21. 

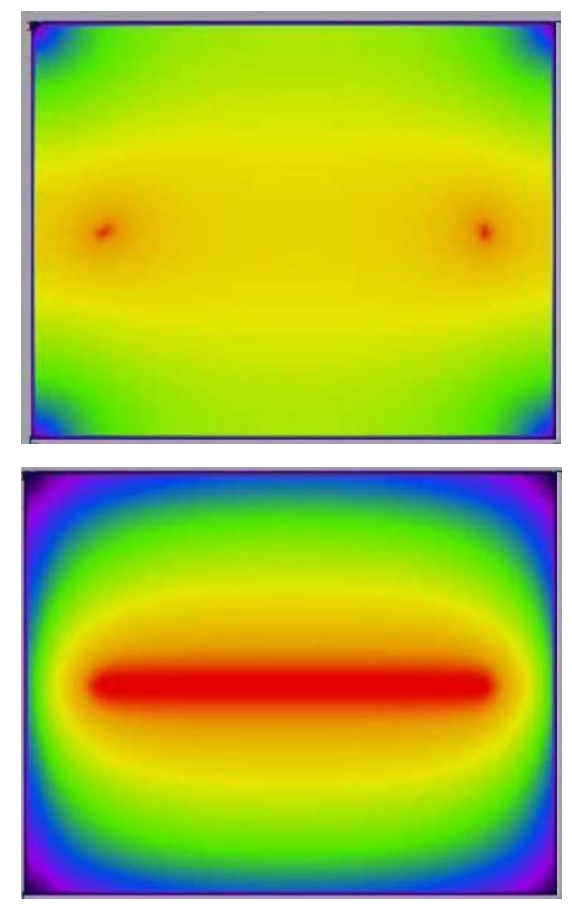

Figure 20. E field distribution inside the TEMt-cell with the septum (upper) and at the location of the interrupted septum (lower)
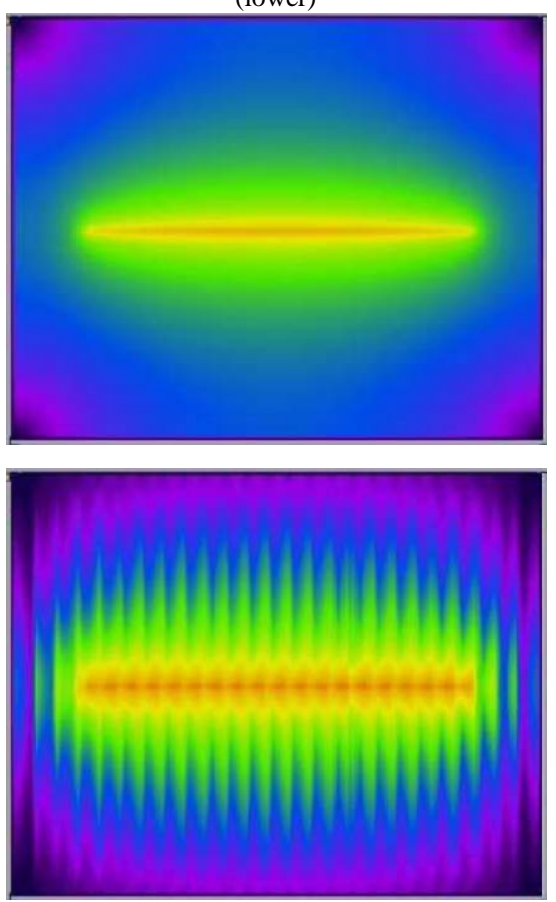

Figure 21. E field distribution just beyond two samples, at a position with interrupted septum: isotropic, homogeneous material (upper) anisotropic, wires at $4 \mathrm{~mm}$ distance (lower)

Both pictures show that a non-homogeneous attenuation is occurring for both samples, so that no equal attenuation may be assumed over the entire cross section of the TEMt-cell

\section{CONCLUSIONS}

In this paper, the measurement of the shielding effectiveness of anisotropic materials has been analyzed and discussed. It turns out that using different measuring setups, different results are obtained, at least in the lower frequency range, where appropriate fixtures are used, allowing small samples of materials.

This is especially the case for the standardized ASTM D4935 cell, and related fixtures based on a TEM mode propagation inside the testcell.

It has been found that the mutual orientation between the (E) field polarization and the direction of the anisotropic structures is the dominant factor influencing the resulting SE value.

At microwave frequencies, where horn antennas are used as measuring probes, the dependency from field polarization with respect to the anisotropic character of the materials is clearly discriminated.

As a general conclusion, it must be stated that more work has to be done on the SE characterisation of anisotropic materials in the lower frequency range, with special attention to the inappropriate use of well established standards.

The formation of an IEEE Standards Association ad hoc working group on this subject should be considered.

\section{REFERENCES}

[1] IEEE Std. 299 - 2006, "IEEE Standard Method for Measuring the Effectiveness of Electromagnetic Shielding Enclsosures", IEEE Febr. 2007

[2] ASTM D4938, "Standard Test Method for Measuring the Electromagnetic Shielding Effectiveness of Planar Materials"

[3] J. Catrysse, "New Test Cell for the Characterization of shielding materials in the far field", IEE Conf. No.326, 1990

[4] E. Hariya et al., "KEC: Instruments to measure the Electromagnetic Shielding Effectiveness", Proc. Int. Symposium on EMC, Tokyo, October 1984

[5] M.S. Sarto et al., "Shielding Effectiveness of Protective Metallic Wire Meshes: EM Modelling and Validation", IEEE Trans. on EMC, Vol. 56, 3, June 2014

[6] A. Tamburrano et al., "Coaxial Waveguide Methods for Shielding Effectiveness Measurement of Planar Materials up to 18 GHz", IEEE Trans. on EMC, Vol. 56, 6, December 2014

[7] NIST Technical Note 1326 - 1989, "Theory and Measurements of Radiated Emissions Using a TEM Cell"

[8] N. Marcuvitz editor, "Waveguide Handbook", Dover Publications 1965 (reprinted from MdGraw Hill 1951)

[9] J. Catrysse, "Shielding Effectiveness of flat samples and conductive gaskets: new measuring cell for the frequency range 1-18 GHz", Proc. EMC Europe 2008 Symposium, Hamburg, Sept. 2008 